\title{
The Feasibility of Laparoscopic Surgery Compared to Open Surgery in Patients with T4 Colorectal Cancer Staged by Preoperative Computed Tomography
}

\author{
Youn-Ju Lee, M.D., Kyung-Ha Lee, M.D., Ji-Yeon Kim, M.D., Jin-Su Kim, M.D. \\ Department of Surgery, School of Medicine, Chungnam National University, Daejeon, Korea
}

\begin{abstract}
Purpose: Decision of laparoscopic surgery (LS) for locally advanced colorectal cancer is based on preoperative computed tomography (CT), notwithstanding the inaccuracy of $\mathrm{T}$ staging. The aim of this study was to compare the differences between LS and open surgery (OS) in their R0 resection rate, short-term results, and oncologic outcomes for T4 colorectal cancer staged by preoperative CT scanning.
\end{abstract}

Methods: A total of 101 patients who had undergone LS were compared with 46 patients who had undergone OS. Preoperative CT scans for all patients indicated T4. Emergency operation, distant metastases, chemoradiotherapy, and multivisceral resection were excluded from the study.

Results: The OS group showed a higher percentage of right-side colon cancer $(p=0.028)$, larger tumor $(p=0.039)$, and postoperative complication rate $(p=0.002)$. There was no difference in $\mathrm{R} 0$ resection rate between the two groups (94.1\% vs. 95.7\%; $p=1.000)$. The LS group showed significantly shorter hospital stays $(p=0.001)$, but a similar operation time $(p=0.103)$ compared with the OS group. No significant difference in five-year overall survival, disease-free survival, and local recurrence was observed between the two groups.

Conclusion: The results of this study show that LS is feasible in the majority of patients with T4 colorectal cancer staged by CT scanning if multivisceral resection is not planned.

Keywords: Laparoscopy, Colorectal neoplasms, Colonic neoplasms, X-ray computed tomography
This is an Open Access article distributed under the terms of the Creative Commons Attribution Non-Commercial License (http:/l creativecommons.org/licenses/by-nc/4.0/) which permits unrestricted non-commercial use, distribution, and reproduction in any medium, provided the original work is properly cited.

\author{
Received October 2, 2015 \\ Revised 1st October 21, 2015 \\ 2nd October 27, 2015 \\ Accepted October 28, 2015 \\ Corresponding author \\ Jin-Su Kim \\ Department of Surgery, School \\ of Medicine, Chungnam National \\ University, 228 Munhwa-ro, Jung- \\ gu, Daejeon 35015, Korea \\ Tel: +82-42-280-8383 \\ Fax: +82-42-257-8024 \\ E-mail: jskim7562@gmail.com
}

Copyright $\odot 2016$ The Journal of Minimally Invasive Surgery. All rights reserved.

\section{INTRODUCTION}

Colorectal cancer, 140,000 new cases of which are diagnosed every year, is the third commonest cancer in the United States. ${ }^{1}$ Early detection and proper treatment are pivotal to a good prognosis. Colorectal cancer prognosis, as reflects the importance of early detection, is directly related to the stage at the time of diagnosis. Most colorectal cancer patients undergo preoperative staging with abdomino-pelvic computed tomography (CT) scanning and work-up to determine the presence of distant metastases. Previous studies have demonstrated, however, that the accuracy of CT scanning for detection of the $\mathrm{T}$ and $\mathrm{N}$ stages ranges from only 60 to $75 \%$. $^{2-4}$

Recently, laparoscopic surgery (LS) has been accepted for the treatment of colorectal cancer worldwide, its oncologic safety and clinical advantage having been proven in prospective multicenter randomized clinical trials. ${ }^{5-10}$ Meanwhile, the criteria for the employment of LS in the treatment of colorectal cancer are developing and evolving, and now include the advanced stage of disease. ${ }^{11,12}$ 
However, the laparoscopic approach has several limitations such as a restricted surgical view, prolonged operative time, and difficulty of en bloc resection for locally advanced colorectal cancers. ${ }^{13,14}$ In light of the challenging aspects and overall difficulty of LS, major surgical societies' guideline have recommended consideration of open surgical resection for locally advanced colorectal cancer if a laparoscopic en bloc resection cannot be performed adequately. In addition, there is a lack of evidence that laparoscopic resection of T4 colorectal cancers are safe and feasible. As already noted, the choice of LS for the treatment of locally advanced colorectal cancer is based on preoperative $\mathrm{CT}$ scanning, the $\mathrm{T}$ staging of which is notoriously inaccurate. Given this unreliability, surgeons naturally are hesitant to follow through with LS for the demanding and troublesome T4 resection procedure.

We hypothesized that not all radiologic T4 colorectal cancers are correlated with pathologic T4 tumors. However, in selected patients, LS offers a clinical advantage such as short hospital stay and similar oncologic safety. Therefore, the aim of this study was to compare R0 resection rate, short-term clinical results, and oncologic outcomes between LS and open surgery (OS) for radiologic T4 colorectal cancer.

\section{MATERIALS AND METHODS}

Prospective database records of 1,312 consecutive patients who had undergone major resection of colorectal cancer between March 2006 and June 2013 were reviewed at our institute. T4 cancer was diagnosed radiologically in 246 patients. Of these patients, emergency operation $(n=43)$, distant metastases $(n=18)$, preoperative chemoradiotherapy $(n=13)$, and multivisceral resection $(n=25)$ were excluded from the study

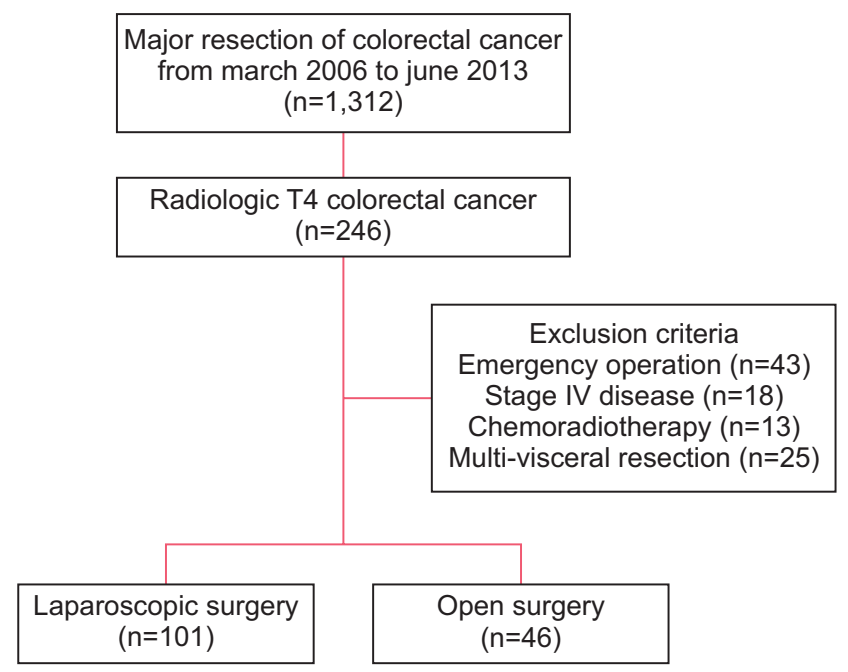

Fig. 1. Study flowchart.
(Fig. 1). One-hundred one LS patients were compared with 46 OS patients over the same period. All of the operations had been performed by two surgeons (J. S. K. and J. Y. K.) experienced in the use of both LS and OS techniques for the treatment of colorectal cancer. This study was reviewed and approved by our institutional review board.

The preoperative work-up included a complete historytaking, a physical examination, complete blood count, liver function test, colonoscopy, CT scanning, and positron emission tomography (PET) scanning. In the cases of rectal cancer, an endorectal ultrasound, pelvic magnetic resonance imaging (MRI), or both were additionally performed to assess the extent of local tumor invasion.

Curative surgery was performed after mechanical bowel preparation. All of the patients received surgical treatment on the principle of excision of the primary tumor. In the LS cases, a inferior-lateral approach which performed by pancreas head exposure after dissection of mesocolon and ligation of central vessels with extracorporeal stapled anastomosis was followed in right-sided colon resection, whereas in left-sided colon resection or rectal resection, medial-to-lateral dissection with intracorporeal double-stapled anastomosis or hand-sewn coloanal anastomosis was performed. The completeness of resection was defined based on the operative and pathology reports and classified as follows: R0 (negative gross and microscopic margins), R1 (negative gross margin with positive microscopic margins), R2 (positive gross margins). Margin involvement was determined by the presence of tumor cells from the outermost margin of the lesion to the surgical plane, or when the maximum distance between the tumor and surgical plane was less than $1 \mathrm{~mm}$.

Tumors were staged, based on the final pathologic reports, according to the American Joint Committee on Cancer's $7^{\text {th }}$ TNM edition. ${ }^{15}$ Specimens were evaluated for tumor size, differentiation, depth of penetration, lymph node metastases, and resection margin. The patients were followed-up on at 4-month intervals for the first 2 years, 6-month intervals for the next 3 years, and annually thereafter. The followups included regular history-taking, physical examination, serum carcinoembryonic antigen (CEA) levels, chest X-ray, abdominopelvic CT scanning, colonoscopy, and PET scanning if available. Recurrence was determined clinical and radiological examinations or by histological confirmation.

A statistical analysis was performed using SPSS (version 20.0 for Windows; SPSS Inc., Chicago, IL). A two-tailed $t$ test for continuous variables and a chi-squared test and/or Fisher's exact test for categorical variables were utilized for comparison of the differences between the two groups. Survival rates were calculated using the Kaplan-Meier method, and the survival curves were compared using a log-rank test. A $p$ value less 
Table 1. Comparison of clinical and pathologic variables between laparoscopic surgery and open surgery

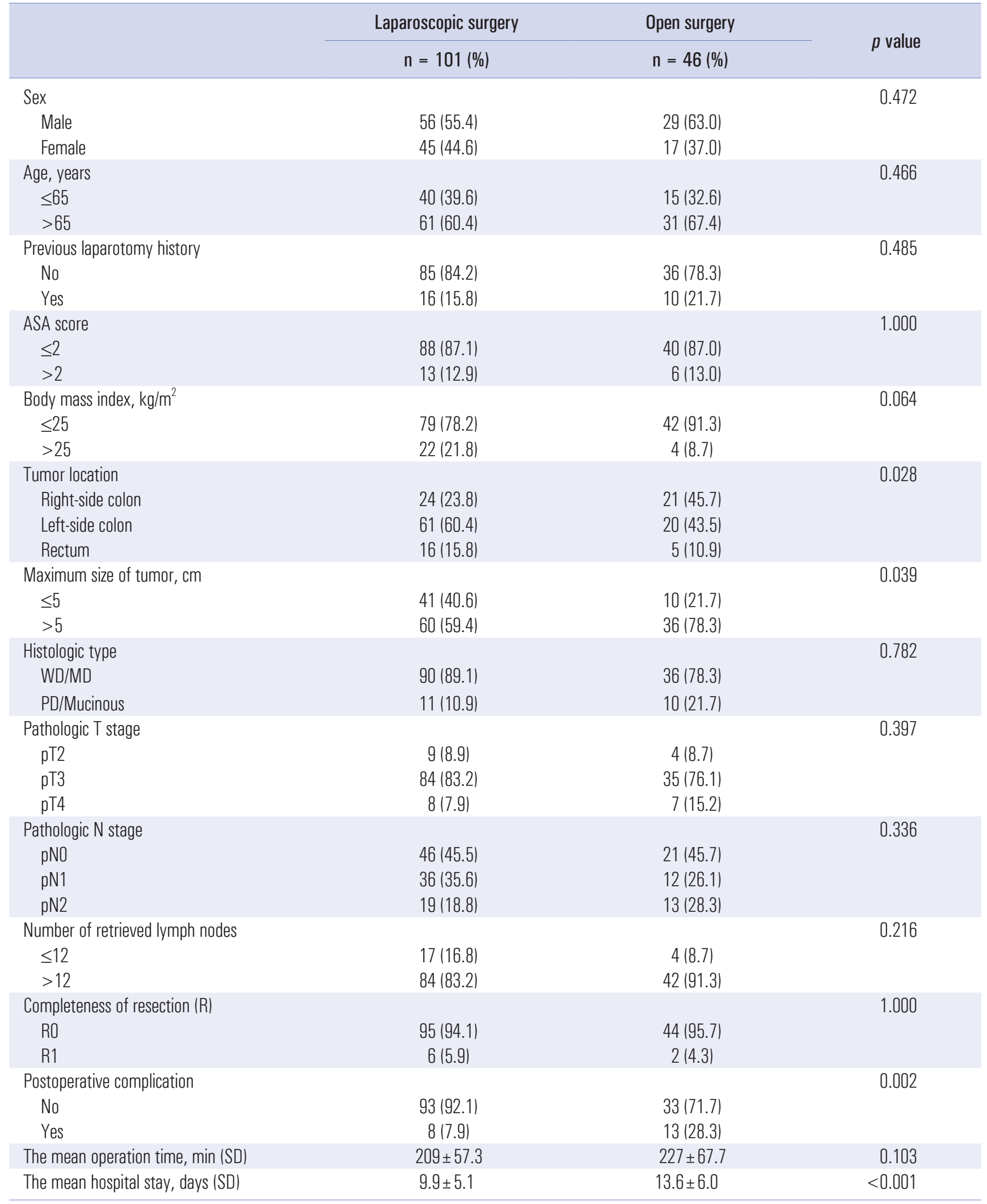

$\mathrm{ASA}=$ American Society of Anesthesiologists; $\mathrm{WD}=$ well differentiated; $\mathrm{MD}=$ moderately differentiated; $\mathrm{PD}=$ poorly differentiated; $\mathrm{Muc}=$ mucinous differentiated. 
than 0.05 was considered statistically significant.

\section{RESULTS}

Five patients (5.0\%) who initially underwent the LS were converted to the OS due to duodenal invasion during the right hemicolectomy procedure $(\mathrm{n}=1)$, uncontrolled bleeding $(\mathrm{n}=2)$, or tumor infiltration into the pelvic sidewall in cases of rectal cancer $(n=2)$. These cases were assigned to the OS group because the aim of this study was to compare clinical and oncologic outcomes between LS and OS. A summary of the LS and OS patient demographics is provided in Table 1. The both groups were well matched in terms of gender, age, history of previous abdominal surgery, and American Society of Anesthesiologists score. However, the patients in the OS group showed a higher percentage of right-side colon cancer $(p=0.028)$, larger tumor $(p=0.039)$, and postoperative complication rate $(p=0.002)$. It is noteworthy that groups' respective rates of resection completion were similar (R0 resection rates: $94.1 \%$ in LS group, $95.7 \%$ in OS group;

Table 2. Primary operations

\begin{tabular}{|ccc|}
\hline & Laparoscopic surgery & Open surgery \\
\cline { 2 - 3 } & $\mathrm{n}=101(\%)$ & $\mathrm{n}=46(\%)$ \\
\hline Primary operations & & \\
\hline Right hemicolectomy & $23(22.8)$ & $21(45.7)$ \\
\hline Transverse colectomy & $1(1.0)$ & $2(4.3)$ \\
\hline Left hemicolectomy & $1(1.0)$ & $1(2.2)$ \\
\hline Anterior resection & $38(37.5)$ & $9(19.6)$ \\
\hline Low anterior resection & $34(33.7)$ & $12(26.0)$ \\
\hline Abdominoperineal resection & $2(2.0)$ & 0 \\
\hline Subtotal colectomy & $2(2.0)$ & $1(2.2)$ \\
\hline
\end{tabular}

Table 3. Postoperative complications

\begin{tabular}{lcc} 
& Laparoscopic surgery & Open surgery \\
\cline { 2 - 3 } & $\mathrm{n}=101(\%)$ & $\mathrm{n}=46(\%)$ \\
\hline Wound infection & $2(2.0)$ & $3(6.5)$ \\
Urinary dysfunction & $2(2.0)$ & $3(6.5)$ \\
Postoperative ileus & $1(1.0)$ & $4(8.7)$ \\
Intra-abdominal abscess & $1(1.0)$ & $1(2.2)$ \\
Anastomotic leakage & $1(1.0)$ & $1(2.2)$ \\
pneumonia & $1(1.0)$ & $1(2.2)$ \\
\hline
\end{tabular}

$p=1.000)$. Histologic differentiation $(p=0.782)$, pathologic $\mathrm{T}$ stage $(p=0.397)$, pathologic $\mathrm{N}$ stage $(p=0.336)$, and the numbers of retrieved lymph nodes $(p=0.216)$ were not significantly different. The LS group showed significantly shorter hospital stays $(p<0.001)$, whereas similar operation time $(p=0.103)$ compared with OS group.

As for the surgical procedures and postoperative complications, details are provided in Tables 2 and 3, respectively. Anterior resection (37.5\%) and low anterior resection (33.7\%) were common procedures in the LS group, whereas in the OS group, right hemicolectomy (45.7\%) was the most common procedure (Table 2). There was no postoperative mortality within 30 days of operation. However, 8 LS patients (8.0\%) and 13 OS patients (28.3\%) eventually developed postoperative complications. The total number of complication events significantly differed between the two groups $(p=0.002)$. In the LS group, wound infection was noted in 2 patients $(2.0 \%)$, urinary dysfunction in $2(2.0 \%)$, postoperative ileus in $1(1.0 \%)$, intra-abdominal abscess in 1 (1.0\%), anastomotic leakage in $1(1.0 \%)$, and pneumonia in $1(1.0 \%)$, meanwhile in the OS group, wound infection developed in 3 patients (6.5\%), urinary dysfunction in $3(6.5 \%)$, postoperative ileus in $4(8.7 \%)$, intraabdominal abscess in 1 (2.2\%), anastomotic leakage in 1 (2.2\%), and pneumonia in 2 (2.2\%) (Table 3).

For the median follow-up time of 25.1 months in the LS group (range, 3 81.1) and 23.3 months in the OS group (range, $3 \sim 97.8$ ), the 5-year overall survival and disease-free survival rates were $70.3 \%$ and $76.5 \%$, respectively. The 5 -year overall survival (75.2\% vs. $58.7 \% ; p=0.227$; Fig. 2$)$ and diseasefree survival rate $(79.7 \%$ vs. $69.1 \% ; p=0.142$; Fig. 3 ) was not significantly different in the both LS and OS groups. The 5 -year local recurrence rate was neither significantly different (3.4\% vs. 7.8\%; $p=0.705$; Fig. 4).

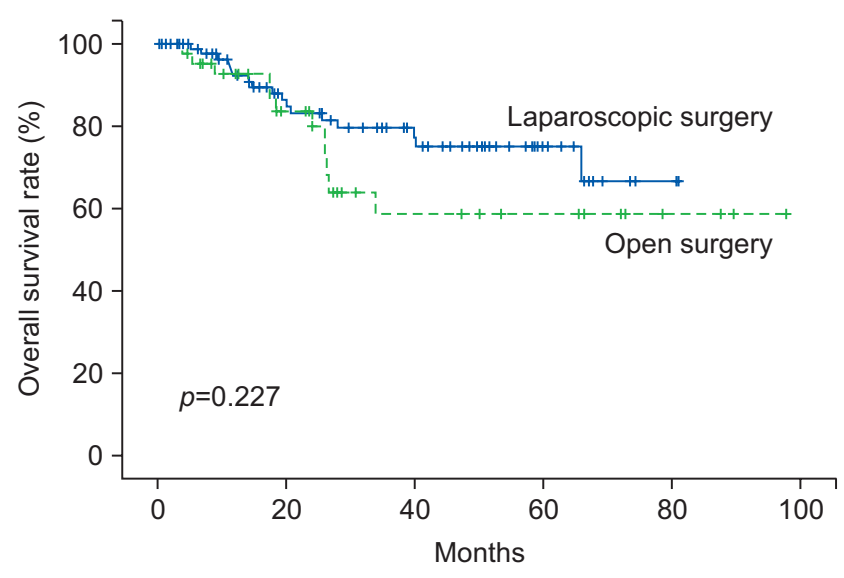

Fig. 2. Overall survival rates for laparoscopic versus open surgery. 


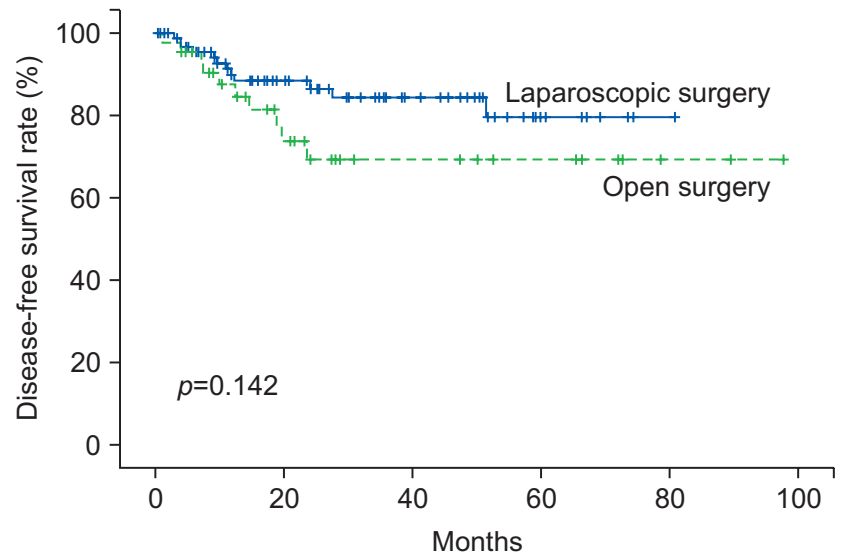

Fig. 3. Disease-free survival rates for laparoscopic versus open surgery.

\section{DISCUSSION}

International surgical societies have not recommended the laparoscopic approach for locally advanced colorectal cancer if a laparoscopic en bloc resection cannot be performed adequately. ${ }^{13,14}$ Even so, the fact that laparoscopic colorectal surgery has potential benefits in short-term outcomes and oncological safety has led surgeons to consider applying this technique to advanced disease. ${ }^{11,12}$ It is well known that the laparoscopic approach to locally advanced colorectal cancer is technically difficult and demanding with high conversion rates ranging from $2.3 \%$ to $26.5 \%$ according to recently published data. ${ }^{11,12,16,17}$ In the present study, the conversion rate was $5.0 \%$, which compares favorably with the earlier results. This low conversion rate can be explained by the exclusion of patients with chemoradiotherapy, bowel perforation, distant metastases, and multi-visceral resection for whom a higher risk of conversion has been reported. ${ }^{18}$

Preoperative CT scanning provides useful information that influences the selection of the clinical treatment strategy. For example, preoperative CT scanning determines resectability whether to operate or not. However, the accuracy of its prediction of $\mathrm{T}$ stage, compared with histology, has ranged from $60 \%$ to $75 \% .^{2-4}$ In our study, of 147 radiologic T4 cancers, only 15 cases (10.2\%) revealed pathologic T4 cancer. This low correlation rate between radiologic and pathologic T4 stage might be attributed to exclusion criteria of emergency operation, chemoradiotherapy, and multivisceral resection. However, relative inability to predict invasion depth complicates the choice of surgical technique. Undoubtedly, surgeons should not have to hesitate to select LS where preoperative CT scanning shows suspected T4 lesion. Still, surgeons should exercise caution when choosing the laparoscopic approach for radiologic T4 cancer, because conversion to OS is correlated with a high rate of postoperative morbidity. ${ }^{9}$

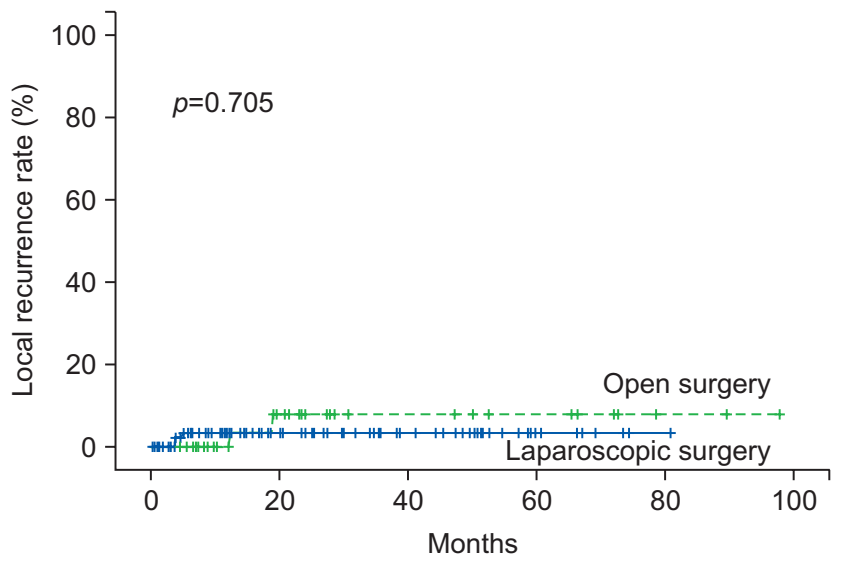

Fig. 4. Local recurrence rates for laparoscopic versus open surgery.

The major findings in our study are that the LS can be achieved low rate of postoperative complication and high rate of R0 resection in patients with radiologic T4 cancer and that this is associated with shorten hospital stay and acceptable oncologic outcomes when compared with OS.

The adoption of the laparoscopic approach to radiologic T4 cancer, meanwhile, basically depends on the surgeon's decision after finishing the stage work-up. The present study found that right-sided tumor location $(p=0.028)$ and large tumor size $(>5 \mathrm{~cm} ; p=0.039$ ) were significantly higher in the OS group than in the LS group. These results suggest that the selection of the surgical technique in the surgeon's routine practice might be based on the tumor location and size if preoperative CT scanning showed T4 tumor and multivisceral resection was not planned. Complex anatomy and the need for careful dissection and ligation of vessels around critical anatomic structure, especially around superior mesenteric vein and artery, may lead a surgeon to avoid laparoscopic approach in patients with right-sided and huge size colon cancer.

En bloc resection of tumor is very important in cases of multivisceral involvement of colorectal cancer; R0 resection, for example, increases survival rate and decreases recurrence. Whereas some surgeons tend to avoid LS when T4 colorectal cancer is demonstrated by preoperative CT scanning, in the present study, R0 resection rate did not significantly differ between the two procedures, though multivisceral resection was excluded (Table 1). Therefore, we considered that the laparoscopic procedure could be performed safely in the selected cases which radiologic T4 was presented.

Three authoritative multicenter randomized trials demonstrated no statistical difference in postoperative complications between LS and OS. ${ }^{5,79}$ In our study, however, there were more complications in the OS group than in the LS group (Table 3). Lacy et al., ${ }^{6}$ correspondingly, also noted a greater number of complications in patients treated by OS than by LS. It seems, 
therefore, that the above noted multicenter randomized trials, though generally considered to be authoritative, should not be assumed to be definitive with respect to the issue of complications.

Concerning long-term results, three of the large-scale studies above mentioned reported 3-year overall survival rates ranging from $67.8 \%$ to $86 \%$ and 3-year disease-free survival rates ranging from $66.8 \%$ to $85 \%$ for all stages. ${ }^{7,80}$ Our present survival data is comparable (70.3\% for overall survival, $76.5 \%$ for disease-free survival) though all of the patients showed T4 lesion by preoperative CT scanning. These favorable oncologic outcomes compared with multicenter randomized trials might be attributable to fact that our study showed low rate of pathologic T4 lesion (10.2\%) and high rate of node negative colorectal cancer (46.0\%).

Previously mentioned multicenter randomized trials showed no statistical differences in the overall survival and diseasefree survival rates between their LS and OS groups, which included patients with any stage of cancer. The present study was also showed that the overall survival and diseasefree survival rates between LS and OS groups were not significantly different. The local recurrence rate for the LS group was comparable to the result obtained in the OS group. Nonetheless, Lacy et al. ${ }^{6}$ found that an LS group of patients had a better overall survival rate $(p=0.02)$ as well as a better cancer-related survival rate $(p=0.006)$ than an OS group with stage III tumor. Guerrieri et al. ${ }^{11}$, evaluating patients with pathologic stage III colon cancer, documented significantly lower local recurrence $(p<0.001)$ and cancer-related death $(p=0.001)$ rates for their LS group relative to their OS group. We as yet do not know the exact mechanism by which LS is associated with better oncologic outcomes than OS in the locally advanced colorectal cancer. We assume that the adoption of laparoscopic approach to radiologic T4 colorectal cancer might be beneficial for certain patient, on this basis, we believe that surgeons should continue trying to utilize LS in the treatment of such patients.

This study is limited by its retrospective design and by the small number of sample size. Selection of surgical technique between LS and OS depended on surgeon's decision. Therefore, there was surgeon's bias in our study and inadequate statistical power because of relatively small study population.

In conclusion, the results of this study show that LS is feasible in the majority of patients with radiologic T4 colorectal cancer if multivisceral resection is not planned. Notwithstanding the limitations of our study, specifically its retrospective nonrandomization and the selection bias resulting from the exercise of the surgeon's preference, we conclude that LS in patients with $\mathrm{T} 4$ colorectal cancer staged by preoperative $\mathrm{CT}$ scanning is technically safe and feasible in selected cases, and has better perioperative results without compromising oncologic outcomes. However, according to our analysis, the likelihood of T4 colorectal cancer, as presented by preoperative CT scanning, was not directly related to actual histologic $\mathrm{T} 4$ stage. Patients with radiologic T4 colorectal cancer should not be excluded from a laparoscopic approach.

\section{REFERENCES}

1) Siegel R, Desantis C, Jemal A. Colorectal cancer statistics, 2014. CA Cancer J Clin 2014;64:104-117.

2) Smith NJ, Bees N, Barbachano Y, Norman AR, Swift RI, Brown G. Preoperative computed tomography staging of nonmetastatic colon cancer predicts outcome: implications for clinical trials. Br J Cancer 2007;96:1030-1036.

3) Dighe S, Blake H, Koh MD, et al. Accuracy of multidetector computed tomography in identifying poor prognostic factors in colonic cancer. Br J Surg 2010;97:1407-1415.

4) Hundt $W$, Braunschweig R, Reiser M. Evaluation of spiral CT in staging of colon and rectum carcinoma. Eur Radiol 1999;9:78-84.

5) Veldkamp R, Kuhry E, Hop WC, et al. Laparoscopic surgery versus open surgery for colon cancer: short-term outcomes of a randomised trial. Lancet Oncol 2005;6:477-484.

6) Lacy AM, Garcia-Valdecasas JC, Delgado S, et al. Laparoscopyassisted colectomy versus open colectomy for treatment of nonmetastatic colon cancer: a randomised trial. Lancet 2002;359:22242229.

7) A comparison of laparoscopically assisted and open colectomy for colon cancer. N Engl J Med 2004;350:2050-2059.

8) Jayne DG, Guillou PJ, Thorpe H, et al. Randomized trial of laparoscopic-assisted resection of colorectal carcinoma: 3-year results of the UK MRC CLASICC Trial Group. J Clin Oncol 2007;25:3061-3068.

9) Guillou PJ, Quirke P, Thorpe H, et al. Short-term endpoints of conventional versus laparoscopic-assisted surgery in patients with colorectal cancer (MRC CLASICC trial): multicentre, randomised controlled trial. Lancet 2005;365:1718-1726.

10) Buunen M, Veldkamp R, Hop WC, et al. Survival after laparoscopic surgery versus open surgery for colon cancer: long-term outcome of a randomised clinical trial. Lancet Oncol 2009;10:4452.

11) Guerrieri M, Campagnacci R, De Sanctis A, et al. Laparoscopic versus open colectomy for TNM stage III colon cancer: results of a prospective multicenter study in Italy. Surg Today 2012;42:10711077.

12) Allaix ME, Degiuli M, Giraudo G, Marano A, Morino M. Laparoscopic versus open colorectal resections in patients with symptomatic stage IV colorectal cancer. Surg Endosc 2012;26:2609-2616.

13) Zerey M, Hawver LM, Awad Z, Stefanidis D, Richardson W, Fanelli RD. SAGES evidence-based guidelines for the laparo- 
scopic resection of curable colon and rectal cancer. Surg Endosc 2013;27:1-10

14) Veldkamp R, Gholghesaei M, Bonjer HJ, et al. Laparoscopic resection of colon Cancer: consensus of the European Association of Endoscopic Surgery (EAES). Surg Endosc 2004;18:1163-1185.

15) Edge SB, Compton CC. The American Joint Committee on Cancer: the 7th edition of the AJCC cancer staging manual and the future of TNM. Ann Surg Oncol 2010;17:1471-1474.

16) Kim KY, Hwang DW, Park YK, Lee HS. A single surgeon's experience with 54 consecutive cases of multivisceral resection for locally advanced primary colorectal cancer: can the laparoscopic approach be performed safely? Surg Endosc 2012;26:493-500.

17) Bretagnol F, Dedieu A, Zappa M, Guedj N, Ferron M, Panis Y. T4 colorectal cancer: is laparoscopic resection contraindicated? Colorectal Dis 2011;13:138-143.

18) Darakhshan A, Lin BP, Chan C, Chapuis PH, Dent OF, Bokey L. Correlates and outcomes of tumor adherence in resected colonic and rectal cancers. Ann Surg 2008;247:650-658. 